\title{
Evaluación del primer nivel de atención para el control de la tuberculosis en Buenos Aires, Argentina
}

\author{
Javier Mariani, Daniel Ferrante², Gabriel Battistella³, Martín Langsam¹, Freddy Pérez y Alejandro \\ Macchia $^{1}$
}

Forma de citar

Mariani J, Ferrante D, Battistella G, Langsam M, Pérez F, Macchia A. Evaluación del primer nivel de atención para el control de la tuberculosis en Buenos Aires, Argentina. Rev Panam Salud Publica. 2020;44:e156. https://doi.org/10.26633/RPSP.2020.156

RESUMEN

Objetivo. Evaluar las funciones de la atención primaria de salud desde la perspectiva de los pacientes con tuberculosis (TB) provenientes de villas de emergencia de la Ciudad Autónoma de Buenos Aires, Argentina. Métodos. Estudio observacional transversal con pacientes adultos con TB y sin TB (NoTB), residentes en villas de emergencia (V) y fuera de ellas (NoV). La percepción de los participantes se evaluó mediante el cuestionario Primary Care Assessment Tool-usuarios (versión abreviada) que mide cuatro dimensiones principales (primer contacto, longitudinalidad de los cuidados, coordinación entre servicios e integralidad) y algunas secundarias. Se utilizó una escala de Likert, desde "No, en absoluto" (1 punto) hasta "Sí, sin duda" (4 puntos). Puntajes $\geq 3$ se consideraron como cumplimiento adecuado de las funciones. Se calcularon promedios para cada dominio y dos puntajes globales: con y sin dominios secundarios.

Resultados. Se incluyeron 83 participantes (20 TB-V, 21 TB-NV, 19 NoTB-V y 23 NoTB-NV). Las funciones evaluadas se percibieron como no adecuadas. El grupo TB-V tuvo los puntajes globales más bajos y en ningún dominio alcanzó 3 puntos. No hubo diferencias significativas en los dominios ni los puntajes globales entre grupos. Los participantes con TB dieron menores puntajes en todos los dominios, excepto en enfoque familiar que fue significativamente mayor que los de los participantes NoTB; el puntaje global sin dominios secundarios fue menor en los participantes con TB que en los NoTB.

Conclusiones. Según la percepción de los participantes con TB y sin TB, las funciones de la atención primaria de salud no son satisfactorias, tanto dentro de las villas de emergencia como fuera de ellas.

Palabras clave Áreas de pobreza; tuberculosis; calidad de la atención de salud; atención primaria de la salud; Argentina.

La Organización Mundial de la Salud propone en las Metas para el Desarrollo Sostenible una reducción de $80 \%$ en la incidencia de tuberculosis (TB) y de $90 \%$ en la mortalidad por esa causa para el año 2030 (1). Por su parte, la Estrategia Fin a la Tuberculosis ha establecido como objetivo reducir ambos indicadores en 90\% y 95\%, respectivamente, entre el año 2015 y el 2035 (2, 3). Para lograr los objetivos planteados, esa estrategia se basa en tres pilares fundamentales, el primero de los cuales es la atención y la prevención integradas y centradas en el paciente, y contempla entre sus principios la adaptación de las intervenciones al contexto único de la epidemia y el entorno sanitario (2).

En Argentina, la implementación de esta estrategia comprende una fuerte participación del primer nivel de atención para desarrollar diversas actividades de prevención, registro, diagnóstico, tratamiento y seguimiento a nivel comunitario (4).

\footnotetext{
Fundación GESICA, Buenos Aires, Argentina. $\square$ Javier Mariani, ja_mariani@hotmail.com

2 Secretaría de Planificación Sanitaria y Gestión en Red, Ministerio de Salud de la Ciudad Autónoma de Buenos Aires, Buenos Aires, Argentina.
}

\footnotetext{
Secretaría de Atención Primaria, Ambulatoria y Comunitaria, Ministerio de Salud de la Ciudad Autónoma de Buenos Aires, Buenos Aires, Argentina.

4 Departamento de Enfermedades Transmisibles y Determinantes Ambientales de la Salud, Organización Panamericana de la Salud, Washington, DC, Estados Unidos de América.
} 
Esto implica un gran desafío, vinculado a las limitaciones del sistema sanitario argentino y a las características sociales, económicas y demográficas de las poblaciones más afectadas por la TB (5)

Desde el año 2013, en Argentina se ha documentado un incremento en el número y en la tasa de incidencia anual de casos de TB (5). La distribución de la enfermedad se concentra en centros urbanos y suburbanos y, dentro de ellos, en grupos socioeconómicamente vulnerables, entre los cuales las personas que habitan en asentamientos irregulares, en particular en villas de emergencias, son los más afectados $(5,6)$. Las villas de emergencia son agrupamientos de hogares caracterizados por viviendas precarias construidas en terrenos que no son propiedad de los habitantes y fuera del marco legal (por lo general, terrenos fiscales), acceso inadecuado e insuficiente a los servicios básicos (agua, energía eléctrica, disposición de residuos cloacales) y hacinamiento (7). En la Ciudad Autónoma de Buenos Aires (CABA), la ciudad más poblada de Argentina, el 7,6\% de la población (alrededor de 230000 personas) habita en villas de emergencia (8). Por otra parte, según datos computados entre enero de 2016 y agosto de 2018 , el 26,3\% de todos los casos de TB de la CABA (765 de 2908 casos totales) se concentraba en estas villas, con lo que se configuran áreas de elevada incidencia (elaboración propia con datos del Sistema Nacional de Vigilancia Sanitaria).

En respuesta al incremento observado en el número de casos antes mencionado, en 2017 el gobierno de la CABA implementó el Plan Integrado de Abordaje de la TB —dependiente en gran medida del primer nivel de atención- para el registro, la prevención, el tratamiento y el seguimiento de los pacientes con TB (9). Este programa tiene como objetivos generales el abordaje integral desde los centros de atención primaria, la evaluación y la derivación a neumólogos especialistas en TB (referencias y contrarreferencias desde y hacia el primer nivel de atención) y el incremento de la intersectorialidad, el monitoreo y la capacitación permanente (9). Sin embargo, a pesar de que desde su implementación se registraron algunas mejoras en los indicadores de proceso (la información relacionada con cada caso y el número de casos registrados en los sistemas de vigilancia epidemiológica por el primer nivel de atención), se deben mejorar otros indicadores de resultados, ya que de los 2729 casos notificados en la CABA en el 2018, en el 35\% no se informó la modalidad de tratamiento y solo en el $18 \%(n=323)$ se documentó el uso del tratamiento directamente observado (10). Esta situación demuestra el enorme desafío que constituye la implementación de un programa de base comunitaria para el control de la TB en el grupo poblacional más vulnerable de una gran ciudad.

El objetivo del presente estudio fue evaluar las funciones del primer nivel de atención del sistema público de salud desde la perspectiva de los pacientes con TB y comparar la percepción de los pacientes provenientes de villas de emergencia de la CABA con la de pacientes de otras áreas de la ciudad y de participantes de ambas áreas sin TB.

\section{MATERIALES Y MÉTODOS}

Se realizó un estudio observacional de corte transversal. El presente trabajo es parte de un proyecto mayor en el que se evaluaron las funciones del primer nivel de atención para el tratamiento de la TB desde las perspectivas de los pacientes, los profesionales de la salud y los gestores, y comprendió un componente cuantitativo y uno cualitativo. Aquí se informan los resultados del componente cuantitativo desde la perspectiva de los pacientes.

\section{Participantes}

Se incluyeron personas de más de 18 años de edad que eran tratadas en el primer nivel de atención del sistema público de salud en la CABA y que en el año previo a la inclusión en el estudio tuvieron diagnóstico de TB (por baciloscopia o cultivo positivo de una muestra respiratoria). Como grupo de control se incluyeron pacientes con diagnóstico de asma bronquial o enfermedad pulmonar obstructiva crónica en el último año; se utilizaron estos diagnósticos como control por tratarse de enfermedades respiratorias que requieren controles habituales y no una única atención. Los participantes se identificaron en los centros de salud y acción comunitaria (CeSAC) de la CABA, tanto dentro como fuera de las villas de emergencia. Los CeSAC son centros polivalentes de atención primaria de salud (APS) y cuentan con médicos generalistas y un número variable de especialidades. La muestra del estudio quedó conformada por cuatro grupos de participantes: pacientes con TB residentes en las villas (TB-V), pacientes con TB no residentes en las villas (TB-NoV), pacientes con otras enfermedades respiratorias residentes en las villas (NoTB-V) y pacientes con otras enfermedades respiratorias no residentes en las villas (NoTB-NoV). $\mathrm{Si}$ bien la comparación de interés primario era entre los TB-V y los TB-NoV, se consideró que incluir participantes sin TB permitiría evaluar el efecto de ser portador de una enfermedad potencialmente estigmatizante (11).

\section{Instrumento de evaluación}

Para evaluar la percepción de los pacientes sobre las funciones de primer nivel de atención se utilizan con frecuencia los cuestionarios Primary Care Assessment Tool (PCAT) (12). Los PCAT se diseñaron para evaluar el primer nivel de atención de los sistemas sanitarios a través de sus principales funciones y está constituido por cuatro cuestionarios que capturan cuatro perspectivas: PCAT-usuarios/pacientes, para pacientes y usuarios; PCAT-proveedores, para integrantes del equipo sanitario, incluidos los profesionales de la salud; PCAT-centros, para directores de centros de APS; y PCAT-sistemas, para gestores. Estos cuestionarios se han utilizado para evaluar las funciones de la APS de los sistemas de salud en diversas enfermedades, incluida la TB (13).

La versión abreviada del cuestionario PCAT-pacientes/usuarios que fue utilizada en el presente estudio tiene traducción y adaptación cultural en Argentina y demostró tener adecuadas propiedades psicométricas (14-16). Este instrumento consta de cuatro dominios principales que reflejan las funciones esenciales de la APS: primer contacto, que incluye los subdominios utilización de los servicios de APS (1 pregunta) y acceso a los centros de APS (2 preguntas); longitudinalidad de los cuidados, que incluye los subdominios continuidad de la atención (4 preguntas) y coordinación de la atención (4 preguntas); la coordinación entre los servicios de atención (1 pregunta); y la integralidad, que incluye los subdominios servicios proporcionados (3 preguntas) y servicios disponibles (4 preguntas). Además, contiene dos dominios secundarios que capturan 
otras funciones de la APS: enfoque familiar de la atención (2 preguntas) y orientación comunitaria (1 pregunta). Finalmente, este cuestionario recaba información sobre el género, la edad, la situación laboral, el máximo nivel de instrucción alcanzado y tres preguntas dirigidas a determinar el grado de identificación del entrevistado con un centro de salud o un médico de APS (el reconocimiento de un centro o un profesional como su centro o profesional tratante ante cualquier problema de salud).

Cada dominio del cuestionario está construido con preguntas que se responden mediante una escala de Likert de 1 a 4 ("No, en absoluto"; "Probablemente no"; "Probablemente sí"; "Sí, sin duda"), además de "No sé". Para analizar las respuestas se sumaron los puntajes dentro de cada dominio; las respuestas "No sé" se computaron como 2 (16). El valor global se calculó de dos formas: como el puntaje promedio de todos los dominios y el de solo los dominios primarios. Se consideró que un puntaje de 3 o más indica la percepción de una adecuada orientación del sistema hacia la APS (17).

\section{Procedimientos}

Se realizó un muestreo por conveniencia con los posibles participantes accesibles a los investigadores.

La invitación a los pacientes para participar en el estudio la realizaron los profesionales de los CeSAC durante las consultas en el centro. En caso de aceptar participar, se concertó una entrevista con los investigadores del estudio en la que se les explicaron los objetivos y se les solicitó firmar el consentimiento informado escrito. Posteriormente, los investigadores aplicaron el cuestionario PCAT-usuarios abreviado a todos los participantes. Todas las entrevistas se realizaron de manera presencial y en privado, en los CeSAC a los que concurrían habitualmente los participantes.

\section{Análisis estadístico}

Se estimó que una muestra de 20 participantes por grupo garantizaría un poder mayor de $90 \%$ para detectar una diferencia entre los grupos de al menos 2 puntos en el puntaje global, considerando una desviación estándar de 1,7 y un error tipo I de $5 \%$, en la comparación de los 4 grupos (18).

Los puntajes de cada dominio se calcularon como la suma de los puntajes dados por cada participante en cada dominio dividida entre el número de preguntas del dominio. Los puntajes globales se calcularon como la suma de todas las respuestas por participante dividida entre el número total de preguntas.

Para describir los datos categóricos se utilizaron frecuencias absolutas y relativas. Los datos continuos se describieron mediante medias y desviaciones estándar (DE) o medianas y rangos intercuartilos (RIQ), según siguieran una distribución normal o no, respectivamente. El supuesto de normalidad se evaluó mediante la prueba de Shapiro-Wilk y la inspección de histogramas y gráficos cuantil-cuantil. Los datos categóricos se compararon entre grupos mediante la prueba de la ji al cuadrado o la prueba exacta de Fisher, según correspondiera. Para comparar los datos continuos entre más de dos grupos se utilizó la prueba de ANOVA o la de Kruskal-Wallis, según el tipo de distribución. Para comparaciones post-hoc se utilizaron valores de $p$ ajustados mediante el método de Bonferroni; este método se utiliza para mantener el error tipo I al nivel deseado en los casos en que se hacen múltiples comparaciones. En las comparaciones de dos grupos, se utilizó la prueba de la $t$ de Student o la de la U de Mann-Whitney, en casos de distribución normal y no normal, respectivamente.

Adicionalmente, se realizaron análisis ajustados por las diferencias en las características demográficas. Para esto se utilizaron modelos de regresión lineal múltiple, con los puntajes como variables dependientes y los grupos como variables independientes; como covariables se utilizaron las variables demográficas que mostraron asociación $(p<0,10)$ en el análisis de regresión lineal simple.

Todos los análisis se realizaron a dos colas y se tomó un valor de $p<0,05$ como estadísticamente significativo. Para los análisis se utilizó el paquete estadístico $R$, versión 3.6.1 para MacOS (19).

\section{Consideraciones éticas}

El estudio fue aprobado por el Comité de Ética en Investigación del Hospital de Agudo Juan P. Fernández (CEI_201910) y por el Comité de Revisión Ética de la Organización Panamericana de la Salud (PAHO-2019-02-0016). Todos los participantes firmaron un consentimiento informado escrito, en el que aceptaron participar en el estudio. Dos autores tuvieron acceso a los datos y responden por la integridad de los mismos. Para mantener el anonimato, los formularios PCAT se completaron de manera anónima y se ingresaron a la base de datos por personas ajenas a las entrevistas, sin identificación de los participantes.

\section{RESULTADOS}

Entre el 19 de junio y el 26 de agosto de 2019 se incluyeron 83 participantes (20 TB-V, 21 TB-NV, 19 NoTB-V y 23 NoTB-NV).

Si bien se observaron diferencias entre los grupos en cuanto a la distribución por edad, género y situación laboral (cuadro 1), estas fueron a expensas de los participantes de los grupos de control sin TB.

La mitad de los participantes con TB eran hombres y tenían una mediana de edad de 33,0 años; el 43,9\% tenía como instrucción máxima la primaria completa o menos; $34,1 \%$ no tenía empleo; y el 61,0\% tenía una fuerte identificación con su centro o su profesional médico de APS.

\section{Percepción de la orientación del sistema hacia la APS}

En el cuadro 2 se puede observar que los puntajes globales del PCAT, tanto de toda la muestra de estudio como de cada uno de los grupos, se encuentran por debajo de 3 , sin diferencias significativas entre los grupos; si se consideran los participantes en su conjunto, ningún dominio individual alcanzó un promedio de 3.

El grupo TB-V tuvo los puntajes globales más bajos, aunque sin diferencias estadísticamente significativas con el resto. Además, en este grupo ningún dominio alcanzó un puntaje de 3 , indicativo de una percepción de adecuado funcionamiento del sistema. Los dominios que alcanzaron valores de 3 puntos o más en los grupos fueron: enfoque familiar en el grupo TB-NoV, longitudinalidad en el grupo NoTB-V y coordinación en el grupo NoTB-NoV. No obstante, no hubo diferencias estadísticamente significativas entre los grupos en ninguno de los dominios individuales ni entre los puntajes globales (figura 1). 
CUADRO 1. Características sociodemográficas de los participantes en el estudio sobre la atención en el primer nivel de salud para el control de la tuberculosis (TB) según el grupo de estudio, Ciudad Autónoma de Buenos Aires, 2019

\begin{tabular}{|c|c|c|c|c|c|c|}
\hline \multirow[b]{2}{*}{ Variable } & \multirow[b]{2}{*}{ Todos los grupos } & \multicolumn{4}{|c|}{ Grupo de estudio } & \multirow[b]{2}{*}{$p$} \\
\hline & & Con TB en las villas ${ }^{a}$ & $\begin{array}{c}\text { Con TB fuera de las } \\
\text { villas }\end{array}$ & $\begin{array}{l}\text { Con otras afecciones } \\
\text { respiratorias en las villas }\end{array}$ & $\begin{array}{l}\text { Con otras afecciones } \\
\text { respiratorias fuera de } \\
\text { las villas }\end{array}$ & \\
\hline Número de participantes & 83 & 20 & 21 & 19 & 23 & \\
\hline Edad, mediana (rango intercuartílico) & $40,0(28,5-52,0)$ & $32,0(21,8-34,0)$ & $37,0(27,0-46,0)$ & $36,0(26,0-47,5)$ & $63,0(53,5-71,5)$ & $<0,001^{c}$ \\
\hline $\begin{array}{l}\text { Género, } n(\%) \\
\text { Hombre } \\
\text { Mujer } \\
\text { Otro }\end{array}$ & $\begin{array}{c}30(36,1) \\
51(61,4) \\
2(2,5)\end{array}$ & $\begin{array}{c}13(65,0) \\
7(35,0) \\
0(0,0)\end{array}$ & $\begin{array}{c}9(42,9) \\
12(57,1) \\
0(0,0)\end{array}$ & $\begin{array}{c}4(21,0) \\
14(73,7) \\
1(5,3)\end{array}$ & $\begin{array}{c}4(17,4) \\
18(78,3) \\
1(4,3)\end{array}$ & $0,010^{d}$ \\
\hline $\begin{array}{l}\text { Escolaridad, } n(\%) \\
\text { Menos que primaria } \\
\text { Primaria completa } \\
\text { Secundaria incompleta } \\
\text { Secundaria completa } \\
\text { Más que secundaria }\end{array}$ & $\begin{array}{l}9(10,8) \\
17(20,5) \\
20(24,1) \\
26(31,3) \\
11(13,3)\end{array}$ & $\begin{array}{l}4(20,0) \\
5(25,0) \\
6(30,0) \\
4(20,0) \\
1(5,0)\end{array}$ & $\begin{array}{l}2(9,5) \\
7(33,3) \\
2(9,5) \\
7(33,3) \\
3(14,4)\end{array}$ & $\begin{array}{l}3(15,8) \\
1(5,2) \\
5(26,3) \\
6(31,6) \\
4(21,1)\end{array}$ & $\begin{array}{l}0(0,0) \\
4(17,4) \\
7(30,4) \\
9(39,2) \\
3(13,0)\end{array}$ & $0,206^{d}$ \\
\hline $\begin{array}{l}\text { Situación laboral, } n(\%) \\
\text { Estudiante } \\
\text { Desempleado } \\
\text { Trabajador } \\
\text { Jubilado }\end{array}$ & $\begin{array}{c}8(9,6) \\
29(34,9) \\
34(41,0) \\
12(14,5)\end{array}$ & $\begin{array}{l}4(20,0) \\
7(35,0) \\
9(45,0) \\
0(0,0)\end{array}$ & $\begin{array}{c}2(9,5) \\
7(33,3) \\
11(52,4) \\
1(4,8)\end{array}$ & $\begin{array}{l}2(10,5) \\
9(47,4) \\
8(42,1) \\
0(0,0)\end{array}$ & $\begin{array}{c}0(0,0) \\
6(26,1) \\
6(26,1) \\
11(47,8)\end{array}$ & $<0,001^{\mathrm{d}}$ \\
\hline $\begin{array}{l}\text { Identificación, } n(\%)^{b} \\
\text { Ninguna } \\
\text { Débil } \\
\text { Fuerte/Muy fuerte }\end{array}$ & $\begin{array}{l}13(15,7) \\
11(13,2) \\
59(71,1)\end{array}$ & $\begin{array}{c}6(30,0) \\
1(5,0) \\
13(65,0)\end{array}$ & $\begin{array}{c}6(28,6) \\
3(14,3) \\
12(57,1)\end{array}$ & $\begin{array}{c}0(0,0) \\
4(21,1) \\
15(78,9)\end{array}$ & $\begin{array}{c}1(4,3) \\
3(13,0) \\
19(82,7)\end{array}$ & $0,029 \mathrm{~d}$ \\
\hline
\end{tabular}

Fuente: elaborado por los autores a partir de los resultados obtenidos.

a Villas de emergencia: agrupamientos de viviendas precarias construidas en terrenos ajenos y fuera del marco legal, acceso inadecuado e insuficiente a los servicios básicos y hacinamiento. - Identificación: grado en que el participante puede identificar a un profesional o centro de atención primaria de salud como su proveedor de referencia ante problemas de salud.

- Los valores de $p$ corresponden a la prueba de ANOVA.

¿

CUADRO 2. Respuestas al cuestionario Primary Care Assessment Tool (PCAT)-usuarios en el estudio sobre la atención en el primer nivel de salud para el control de la tuberculosis (TB) según el grupo de estudio, Ciudad Autónoma de Buenos Aires, 2019

\begin{tabular}{|c|c|c|c|c|c|c|}
\hline \multirow[b]{2}{*}{ Dominio o variable } & \multirow[b]{2}{*}{ Todos los grupos } & \multicolumn{4}{|c|}{ Grupo de estudio } & \multirow[b]{2}{*}{$p^{\mathrm{b}}$} \\
\hline & & Con TB en las villas ${ }^{\mathrm{a}}$ & $\begin{array}{l}\text { Con TB fuera de las } \\
\text { villas }\end{array}$ & $\begin{array}{l}\text { Con otras afecciones } \\
\text { respiratorias en las villas }\end{array}$ & $\begin{array}{l}\text { Con otras afecciones } \\
\text { respiratorias fuera de } \\
\text { las villas }\end{array}$ & \\
\hline Primer contacto, media (DE) & $2,5(0,7)$ & $2,3(0,9)$ & $2,4(0,5)$ & $2,6(0,7)$ & $2,7(0,6)$ & 0,245 \\
\hline Continuidad, media (DE) & $2,8(0,6)$ & $2,6(0,6)$ & $2,9(0,5)$ & $3,0(0,6)$ & $2,8(0,6)$ & 0,133 \\
\hline Coordinación, media (DE) & $2,8(1,0)$ & $2,5(1,0)$ & $2,9(0,9)$ & $2,9(1,1)$ & $3,0(0,9)$ & 0,411 \\
\hline Enfoque familiar, media $(\mathrm{DE})^{c}$ & $2,7(1,2)$ & $2,9(1,3)$ & $3,2(1,1)$ & $2,5(1,2)$ & $2,4(1,2)$ & 0,107 \\
\hline Orientación comunitaria, media $(\mathrm{DE})^{\mathrm{c}}$ & $1,8(1,0)$ & $2,0(1,2)$ & $1,7(0,8)$ & $2,0(1,2)$ & $1,6(0,9)$ & 0,472 \\
\hline PCAT global, media (DE) & $2,7(0,5)$ & $2,6(0,7)$ & $2,6(0,3)$ & $2,8(0,5)$ & $2,8(0,5)$ & 0,566 \\
\hline $\begin{array}{l}\text { PCAT global sin dominios secundarios, } \\
\text { media (DE) }\end{array}$ & $2,7(0,5)$ & $2,6(0,7)$ & $2,6(0,4)$ & $2,8(0,5)$ & $2,9(0,5)$ & 0,187 \\
\hline
\end{tabular}

Fuente: elaborado por los autores a partir de los resultados obtenidos.

Nota: DE: desviación estándar.

a Villas de emergencia: agrupamientos de viviendas precarias construidas en terrenos ajenos y fuera del marco legal, acceso inadecuado e insuficiente a los servicios básicos y hacinamiento.

'Los valores de $p$ corresponden a la prueba de ANOVA.

${ }^{\circ}$ Dominio secundario.

En la figura 2 se observa que menos del $50 \%$ de los participantes, en todos los grupos, tuvieron puntajes globales promedio de 3 o más. No se observaron diferencias significativas en las comparaciones entre los participantes con TB dentro y fuera de las villas (cuadro 3).

Los resultados no cambiaron al ajustar por las diferencias demográficas.

\section{Comparación entre los participantes con TB y sin TB}

En comparación con los participantes sin TB, los que tenían diagnóstico de TB presentaron menores valores en el puntaje global y en la mayoría de los dominios, aunque sin significación estadística (cuadro 4). En el puntaje global sin los dominios secundarios, los participantes con TB tuvieron un promedio 
FIGURA 1. Distribución de los puntajes promedio por dominios principales del cuestionario Primary Care Assessment Tool (PCAT)-usuarios en el estudio sobre la atención en el primer nivel de salud para el control de la tuberculosis (TB), por gruposa, Ciudad Autónoma de Buenos Aires, 2019

A: Dominio primer contacto; B: Dominio longitudinalidad; C: Dominio coordinación; D: Dominio integralidad; E: Puntaje global; F: Puntaje global sin los dominios secundarios ${ }^{c}$
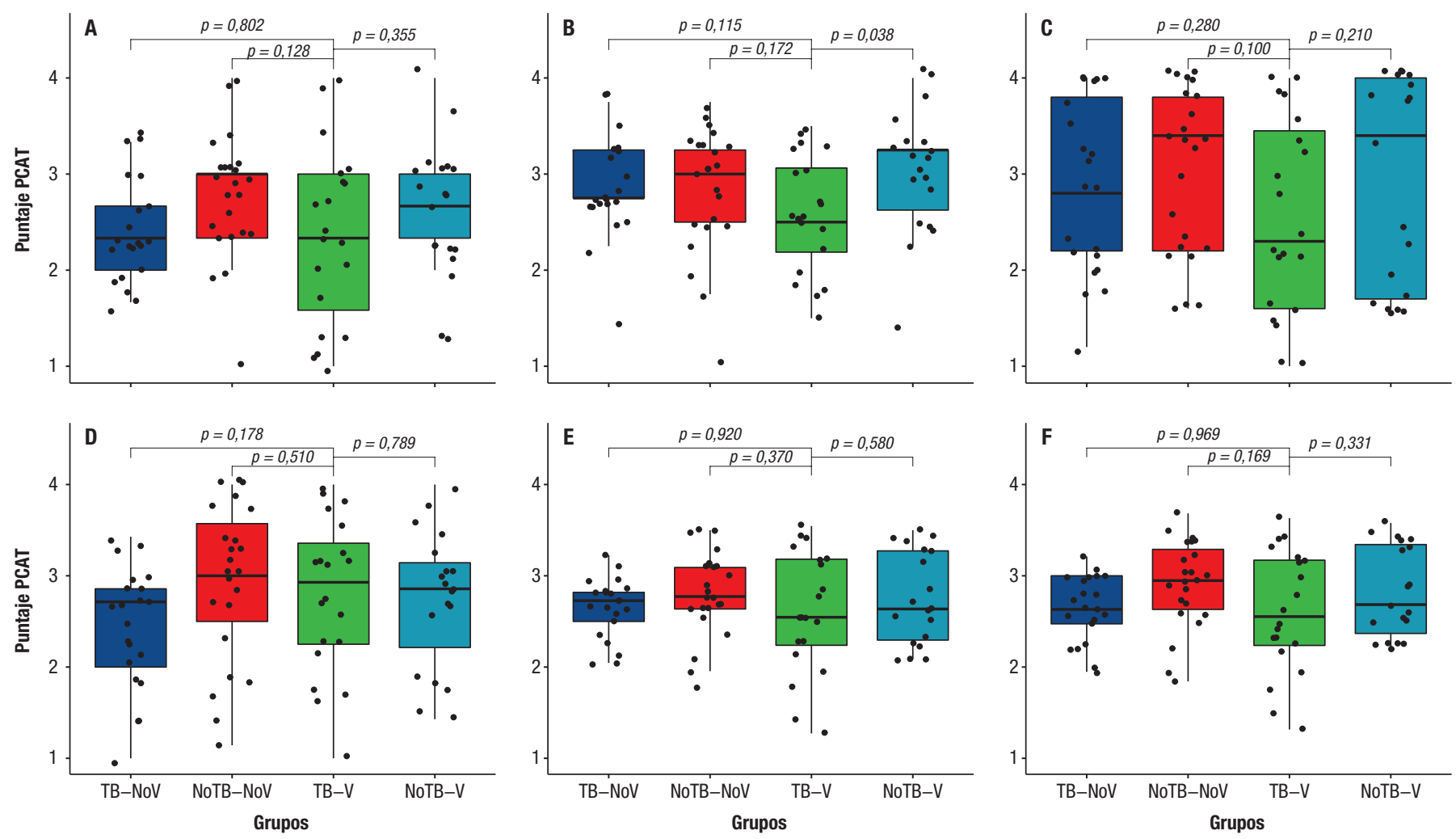

Fuente: elaborado por los autores a partir de los resultados obtenidos.

a TB-V: grupo con TB en las villas; TB-NoV: grupo con TB fuera de las villas; NoTB-V: grupo con otras enfermedades respiratorias en las villas; NoTB-NoV: grupo con otras enfermedades respiratorias fuera de las villas.

${ }^{\mathrm{b}}$ Los valores de $p$ corresponden a la prueba de la $t$ de Student para comparaciones en pares de grupos.

'Los dominios secundarios fueron enfoque familiar y orientación comunitaria.

significativamente menor que los participantes sin TB. Por otra parte, en el dominio enfoque familiar, los participantes con TB tuvieron un promedio significativamente mayor que el de los participantes sin TB. En este análisis, solo el dominio enfoque familiar tuvo un promedio de 3 y se observó en los pacientes con TB (cuadro 4).

\section{DISCUSIÓN}

Los resultados del presente estudio indican que, desde la perspectiva de los usuarios del sistema de salud con TB, las funciones de la APS del sistema de salud no funcionan adecuadamente. Además, esta percepción fue consistente en los diferentes dominios y en los cuatro grupos de pacientes incluidos. Es importante hacer notar que entre los participantes con TB que viven en villas de emergencia, ningún dominio individual ni los puntajes globales alcanzaron el valor mínimo que indicaría un adecuado funcionamiento del primer nivel de atención.

No se han encontrado estudios que evalúen la orientación del sistema sanitario hacia el primer nivel de atención para el cuidado de la TB en poblaciones que habitan en villas de emergencia, por lo que, según nuestro mejor conocimiento, este estudio es el primero en hacerlo desde la perspectiva de los pacientes. En un estudio realizado en cinco ciudades de Brasil, que incluyó pacientes con TB y utilizó una adaptación local del instrumento PCAT para la TB, se informaron resultados no satisfactorios para la región del nordeste (que incluyó dos ciudades) en los dominios primer contacto, integralidad y enfoque familiar, mientras que fueron satisfactorios para longitudinalidad y coordinación (20). Por otra parte, en las tres ciudades del sudeste, los resultados fueron satisfactorios en todos los dominios del cuestionario (20). Este estudio incluyó centros de APS y centros especializados en el tratamiento de la TB (en general, hospitales con recursos humanos y técnicos de alto nivel), sin desglosar los resultados de manera diferenciada, por lo que es difícil establecer una comparación entre esos resultados y los del presente estudio.

Otros estudios realizados en Sudáfrica, Malawi, Hong Kong y Shanghái, que utilizaron el instrumento PCAT para evaluar los sistemas de salud desde la perspectiva de los usuarios, encontraron, al igual que en el presente estudio, valores promedios no satisfactorios para varios dominios (21-23). No obstante, 
FIGURA 2. Frecuencia de los puntajes de aceptación por dominios principales y secundarios ${ }^{a}$ del cuestionario Primary Care Assessment Tool (PCAT)-usuarios en el estudio sobre la atención en el primer nivel de salud para el control de la tuberculosis (TB), por grupos ${ }^{b}$, Ciudad Autónoma de Buenos Aires, 2019c

A: Dominio primer contacto; B: Dominio longitudinalidad; C: Dominio coordinación; D: Dominio integralidad; E: Enfoque familiar; F: Orientación comunitaria; G: Puntaje global; H: Puntaje global sin los dominios secundarios
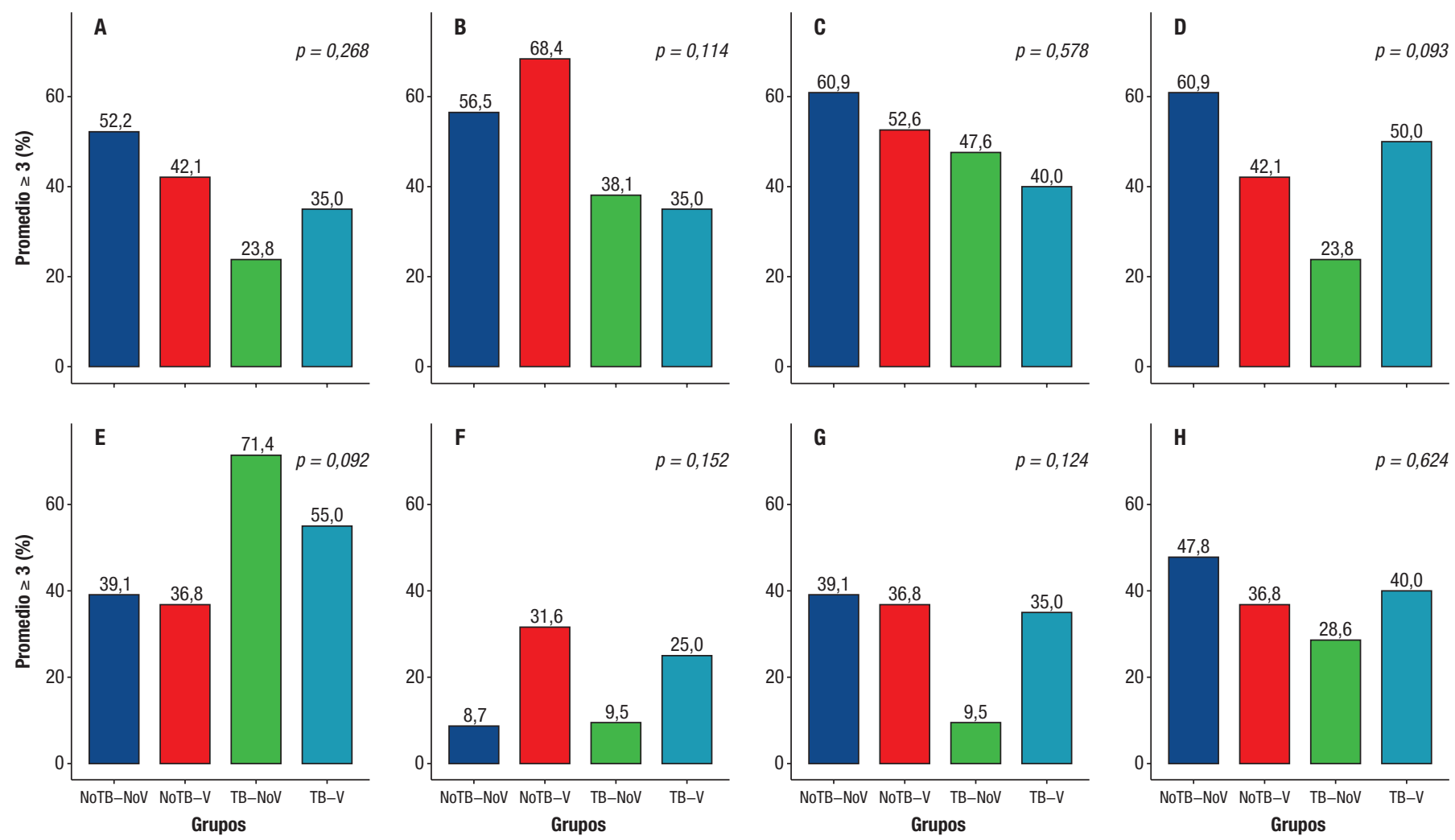

Fuente: elaborado por los autores a partir de los resultados presentados.

a Los dominios secundarios fueron enfoque familiar y orientación comunitaria.

b TB-V: grupo con TB en las villas; TB-NoV: grupo con TB fuera de las villas; NoTB-V: grupo con otras enfermedades respiratorias en las villas; NoTB-NoV: grupo con otras enfermedades respiratorias fuera de las

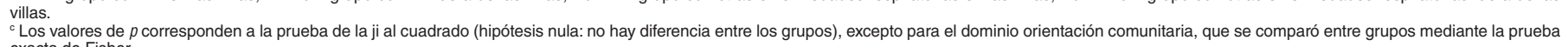
exacta de Fisher.

CUADRO 3. Respuestas al cuestionario Primary Care Assessment Tool (PCAT)-usuarios en el estudio sobre la atención en el primer nivel de salud para el control de la tuberculosis (TB) según el lugar de residencia, Ciudad Autónoma de Buenos Aires, 2019

\begin{tabular}{lccc}
\multicolumn{1}{c}{ Variable } & $\begin{array}{c}\text { Con TB en las } \\
\text { villas }^{\mathrm{a}}\end{array}$ & $\begin{array}{c}\text { Con TB fuera } \\
\text { de las villas }\end{array}$ & $p^{\mathrm{b}}$ \\
Número de participantes & 20 & 21 & \\
Primer contacto, media (DE) & $2,3(0,9)$ & $2,4(0,5)$ & 0,744 \\
Continuidad, media (DE) & $2,6(0,6)$ & $2,9(0,5)$ & 0,105 \\
Coordinación, media (DE) & $2,5(1,0)$ & $2,9(0,9)$ & 0,270 \\
Integralidad, media (DE) $_{\text {Enfoque familiar, media (DE) }}^{\mathrm{c}}$ & $2,8(0,9)$ & $2,4(0,7)$ & 0,170 \\
Orientación comunitaria, media (DE) & $2,9(1,3)$ & $3,2(1,1)$ & 0,367 \\
PCAT global, media (DE) & $2,0(1,2)$ & $1,7(0,8)$ & 0,296 \\
PCAT global sin dominios secundarios, & $2,6(0,7)$ & $2,6(0,3)$ & 0,770 \\
$\quad$ media (DE) & $2,6(0,7)$ & $2,6(0,4)$ & 0,822 \\
\hline
\end{tabular}

Fuente: elaborado por los autores a partir de los resultados obtenidos.

Nota: DE: desviación estándar.

a Villas de emergencia: agrupamientos de viviendas precarias construidas en terrenos ajenos y fuera del marco legal, acceso inadecuado e insuficiente a los servicios básicos y hacinamiento.

- Los valores de $p$ corresponden a la prueba de la $t$ de Student.

Dominio secundario.
CUADRO 4. Respuestas al cuestionario Primary Care Assessment Tool (PCAT)-usuarios en el estudio sobre la atención en el primer nivel de salud para el control de la tuberculosis (TB) según el estado clínico del paciente, Ciudad Autónoma de Buenos Aires, 2019

\begin{tabular}{lccc}
\multicolumn{1}{c}{ Variable } & Con TB & Sin TB & $p^{\mathrm{a}}$ \\
Número de participantes & 41 & 42 & \\
Primer contacto, media (DE) & $2,4(0,7)$ & $2,7(0,7)$ & 0,055 \\
Continuidad, media (DE) & $2,7(0,6)$ & $2,9(0,6)$ & 0,168 \\
Coordinación, media (DE) & $2,7(1,0)$ & $3,0(1,0)$ & 0,222 \\
$\quad$ Integralidad, media (DE) & $2,6(0,8)$ & $2,8(0,8)$ & 0,174 \\
Enfoque familiar, media (DE) & $3,0(1,2)$ & $2,4(1,2)$ & 0,022 \\
$\quad$ Orientación comunitaria, & $1,8(1,0)$ & $1,8(1,1)$ & 0,850 \\
$\quad$ media (DE) & & & \\
PCAT global, media (DE) & $2,6(0,5)$ & $2,8(0,5)$ & 0,443 \\
$\begin{array}{l}\text { PCAT global sin dominios secundarios, } \\
\quad \text { media (DE) }\end{array}$ & $2,6(0,5)$ & $2,9(0,5)$ & 0,033 \\
\hline
\end{tabular}

Fuente: elaborado por los autores a partir de los resultados obtenidos.

Nota: DE, desviación estándar.

Los valores de $p$ corresponden a la prueba de la $t$ de Student.

${ }^{b}$ Dominio secundario. 
ninguno de los estudios citados se focalizó en poblaciones vulnerables con TB.

La TB afecta particularmente a los grupos más pobres y vulnerables, y el fortalecimiento del primer nivel de atención para alcanzar la asistencia sanitaria universal es la mejor estrategia para terminar con la enfermedad $(24,25)$. Esto incluye asegurar el acceso a los servicios de salud de toda la población, priorizar las necesidades de los grupos de mayor necesidad, evitar el gasto de bolsillo en salud con la cobertura completa de fondos públicos e invertir más en el primer nivel de atención (24). La evaluación del sistema sanitario desde la perspectiva de los usuarios es de utilidad para identificar espacios de mejora, particularmente ante las tendencias adversas en la incidencia y la mortalidad por TB que se han observado en Argentina (4).

La percepción de los usuarios acerca del sistema de atención primaria es una parte importante en la evaluación de la calidad de los servicios y permite identificar aspectos específicos que se deben abordar para mejorar los resultados (26). Si bien existen numerosos instrumentos para evaluar la función del primer nivel de atención de los sistemas sanitarios, se utilizó el PCAT por sus adecuadas propiedades psicométricas y por tener validación y adaptación transcultural en Argentina $(14,15,27)$.

El dominio primer contacto, que abarca la utilización de los servicios y el acceso a la consulta en el primer nivel de atención, ha demostrado ser particularmente dificultoso en el caso de la TB y obtuvo el puntaje más bajo en el grupo TB-V (14). En otros contextos, las limitaciones del primer contacto estuvieron vinculadas no solo a la accesibilidad geográfica, sino también a la disponibilidad de consultas con profesionales de la salud adecuadamente capacitados en el manejo de la enfermedad y de las tecnologías necesarias para establecer el diagnóstico de manera oportuna $(14,28)$. De forma similar, los resultados para el resto de los dominios indican que es necesario fortalecer los elementos relacionados con la coordinación, la longitudinalidad en la atención y la integralidad - los servicios que se encuentran disponibles y los que se proporcionan- en la asistencia a los pacientes (atención centrada en el paciente). El enfoque familiar según los pacientes con TB fue adecuado (aunque más alto fuera de las villas de emergencia); esto puede estar relacionado con la búsqueda de contactos convivientes y con la planificación del tratamiento conjuntamente con el de otros integrantes del grupo familiar (29).

\section{Limitaciones}

El presente estudio tiene varias limitaciones que se deben tener en cuenta al momento de interpretar los resultados. La muestra no fue aleatoria, ya que se utilizó un muestreo por conveniencia con pacientes que estaban en contacto con el sistema sanitario y que aceptaron concurrir a una entrevista con un integrante del equipo de investigación; esto puede haber resultado en un grupo de pacientes con una percepción más positiva en relación al sistema de salud. Además, las entrevistas fueron presenciales, lo que podría haber sesgado las respuestas en un sentido favorable al sistema. Sin embargo, en ambos casos, los resultados estarían representando un escenario más optimista, lo que indicaría que los desafíos y las necesidades de mejoras pueden ser incluso mayores. El instrumento utilizado no fue específicamente diseñado para evaluar las funciones de la APS en pacientes con TB. No obstante, se decidió utilizar el PCAT general en su versión abreviada por contar con una adaptación validada localmente y por sus adecuadas propiedades psicométricas, además de su extensa utilización internacional que permite establecer comparaciones e identificar problemáticas comunes a otros contextos. Si bien existe una versión de PCAT adaptada a TB, solo está validada en portugués y no tiene adaptación cultural en Argentina (30).

Otra limitación es el insuficiente poder para detectar pequeñas diferencias entre los grupos. Aunque el tamaño de la muestra por grupo debe garantizar un poder mayor de $90 \%$ para detectar una diferencia entre los grupos de al menos 2 puntos en el puntaje global, un estudio con mayor número de participantes podría identificar diferencias de menor magnitud, pero relevantes desde el punto de vista de la planificación.

Los grupos de participantes difirieron en sus características basales, lo que podría reflejar diferencias en las características de las personas a las que representan. A pesar de que esas diferencias basales podrían explicar los resultados en las percepciones respecto a la APS, los análisis ajustados por factores demográficos no cambiaron los resultados.

\section{CONCLUSIONES}

Los resultados del presente estudio indican que de acuerdo con las percepciones de los participantes con TB y sin TB, las funciones de la APS - como efector del sistema de salud en el cual se centra el Plan de Abordaje Integral de la TB — no se cumplen satisfactoriamente, tanto dentro de las villas de emergencia como fuera de ellas. No se observaron diferencias significativas entre las percepciones dentro y fuera de las villas, lo que destaca la necesidad de abordar ambos contextos para fortalecer las funciones de la APS y con ello mejorar los resultados actuales en los indicadores y acercarnos más a la eliminación de la TB.

Contribución de los autores. JM, DF, GB, ML, FP y AM concibieron el estudio original y planificaron los experimentos. JM, ML y AM recolectaron los datos. JM, FP y AM analizaron los datos. Todos los autores interpretaron los resultados, escribieron y revisaron el manuscrito, y aprobaron la versión final.

Agradecimientos. Los autores agradecen a las doctoras Karin Kopitowski y Mariela Weisbrot por su colaboración y proporcionar la versión adaptada para Argentina del PCAT.

Conflictos de intereses. Los autores declaran no tener conflictos de intereses.

Financiamiento. El estudio recibió financiamiento de un proyecto de la Organización Panamericana de la Salud (ES-SDG PAHO-2019-02-0016). Los patrocinadores no participaron de ninguna manera en el diseño del estudio, la colecta y análisis de los datos, la decisión de publicar este trabajo ni la preparación del manuscrito.

Declaración. Las opiniones expresadas en este artículo son únicamente responsabilidad de los autores y no reflejan necesariamente los criterios ni la política de la Revista Panamericana de Salud Pública / Pan American Journal of Public Health y/o la Organización Panamericana de la Salud. 


\section{REFERENCIAS}

1. United Nations. Transforming our world: the 2030 Agenda for Sustainable Development: Sustainable Development Knowledge Platform. New York: UN; 2015 [citado el 5 de mayo de 2020]. Disponible en: https://sustainabledevelopment.un.org/post2015/ transformingourworld

2. World Health Organization. The End TB Strategy. Geneva: WHO; 2015 [citado el 5 de mayo de 2020]. Disponible en: https://www. who.int/tb/End_TB_brochure.pdf?ua $=1$

3. Uplekar M, Weil D, Lonnroth K, Jaramillo E, Lienhardt C, Dias HM, et al. WHO's new End TB Strategy. Lancet. 2015;385(9979):1799-801. doi: http:/ / doi.org/10.1016/S0140-6736(15)60570-0

4. Ministerio de Salud de la Nación. Plan Estratégico Nacional para el Control de la Tuberculosis en Argentina. Buenos Aires: MSAL; 2019 [citado el 10 de mayo de 2020]. Disponible en: http://www.msal. gob.ar/images/stories/bes/graficos/0000001434cnt-2019-03-28_ plan-estrategico-control-tuberculosis.pdf

5. Ministerio de Salud de la Nación. Boletín sobre tuberculosis en la Argentina. Buenos Aires: MSAL; 2019 [citado el 12 de mayo de 2020]. Disponible en: http://www.msal.gob.ar/images/stories/bes/graficos/0000001436cnt-2019-03_boletin-epidemiologico_tuberculosis. pdf

6. Goldberg A. Factores socioculturales en el proceso asistencial de pacientes con tuberculosis del Instituto Vaccarezza del Hospital Muñiz, 2009. Rev Argent Salud Publica. 2010;1(5):13-21.

7. Ezeh A, Oyebode O, Satterthwaite D, Chen YF, Ndugwa R, Sartori J, et al. The history, geography, and sociology of slums and the health problems of people who live in slums. Lancet. 2017;389(10068):54758. doi: http:/ /doi.org/10.1016/S0140-6736(16)31650-6

8. Gobierno de la Ciudad de Buenos Aires. Porcentaje de viviendas habitadas, hogares y población en villas de emergencia sobre el total de la Ciudad. Años 2006/2018. Buenos Aires: Dirección General de Estadísticas y Censos; 2019 [citado el 15 de mayo de 2020]. Disponible en: https: / / www.estadisticaciudad.gob.ar/eyc/?p=50392

9. Ministerio de Salud de la Ciudad Autónoma de Buenos Aires. Plan integrado de abordaje de la tuberculosis. Bol Epidemiol Semanal. 2018; SE 22 [citado el 12 de mayo de 2020]. Disponible en: https:/ / www.buenosaires.gob.ar/sites/gcaba/files/bes_95_se_22_vf.pdf

10. Ministerio de Salud de la Ciudad Autónoma de Buenos Aires. Vigilancia de la tuberculosis. Bol Epidemiol Semanal. 2019; SE 4 [citado el 16 de mayo de 2020]. Disponible en: https://www.buenosaires. gob.ar/sites/gcaba/files/bes_129_se_4_vf.pdf

11. Furin J, Cox H, Pai M. Tuberculosis. Lancet. 2019;393(10181):1642_ 56. doi: http:/ / doi.org/10.1016/S0140-6736(19)30308-3

12. Shi L, Starfield B, Xu J. Validating the Adult Primary Care Assessment Tool. J Fam Pract. 2001;50(2):161-75.

13. De Sá LD, Scatena LM, Rodrigues RA, Nogueira JA, Silva AO, Villa TC. Gateway to the diagnosis of tuberculosis among elders in Brazilian municipalities. Rev Bras Enferm. 2015;68(3):408-14. doi: http://doi.org/10.1590/0034-7167.2015680313i

14. Vázquez Peña F, Harzheim E, Terrasa S, Berra S. Psychometric validation in Spanish of the Brazilian short version of the Primary Care Assessment Tools-user's questionnaire for the evaluation of the orientation of health systems towards primary care. Aten Primaria. 2017;49(2):69-76. doi: http://doi.org/10.1016/j.aprim.2016.04.007

15. Berra S, Hauser L, Audisio Y, Mántaras J, Nicora V, Oliveira MMC, et al. Validez y fiabilidad de la versión argentina del PCAT-AE para evaluar la atención primaria de salud. Rev Panam Salud Publica. 2013;33(1):30-9.

16. Berra S, Audisio Y, Mántaras J, Nicora V, Mamondi V, Starfield B. Adaptación cultural y al sistema de salud argentino del conjunto de instrumentos para la evaluación de la atención primaria de la salud. Rev Argent Salud Publica. 2011;2(8):6-14.
17. Pesse-Sorensen K, Fuentes-García A, Ilabaca J. Primary Care Assessment Tool applied to primary health care workers from Conchalí, Santiago. Rev Med Chil. 2019;147(3):305-13. doi: http://doi. org /10.4067/S0034-98872019000300305

18. Arakawa T, Arcêncio RA, Scatolin BE, Scatena LM, Ruffino-Netto A, Villa TCS. Accessibility to tuberculosis treatment: assessment of health service performance. Rev Latinoam Enferm. 2011;19(4):994-1002.

19. R: A language and environment for statistical computing. Vienna: R Foundation for Statistical Computing; 2020.

20. Villa TC, Ruffino-Netto A, Scatena LM, Andrade RL, Brunello ME, Nogueira JA, et al. Health services performance for TB treatment in Brazil: a cross-sectional study. BMC Health Serv Res. 2011;11(1):241. doi: http:/ / doi.org/10.1186/1472-6963-11-241

21. Wei X, Li H, Yang N, Wong SY, Owolabi O, Xu J, et al. Comparing quality of public primary care between Hong Kong and Shanghai using validated patient assessment tools. PLoS One. 2015;10(3):e0121269. doi: http://doi.org/10.1371/journal.pone.0121269

22. Mukiapini S, Bresick G, Sayed AR, Le Grange C. Baseline measures of primary health care team functioning and overall primary health care performance at Du Noon Community Health Centre. Afr J Prim Health Care Fam Med. 2018;10(1):a1458. doi: http:/ /doi. org/10.4102/phcfm.v10i1.1458

23. Dullie L, Meland E, Hetlevik Ø, Mildestvedt T, Kasenda S, Kantema $\mathrm{C}$, et al. Performance of primary care in different healthcare facilities: a cross-sectional study of patients' experiences in Southern Malawi. BMJ Open. 2019;9(7):e029579. doi: http://doi.org/10.1136/ bmjopen-2019-029579

24. Ki-Moon B. Building a tuberculosis-free world on a foundation of universal health coverage. Lancet. 2019;393(10178):1268-70. doi: http:/ / doi.org/10.1016/S0140-6736(19)30433-7

25. Reid MJA, Arinaminpathy N, Bloom A, Bloom BR, Boehme C, Chaisson $\mathrm{R}$, et al. Building a tuberculosis-free world: The Lancet Commission on Tuberculosis. Lancet. 2019;393(10178):1331-84. doi: http://doi.org/10.1016/S0140-6736(19)30024-8

26. Macinko J, Guanais FC. Population experiences of primary care in 11 Organization for Economic Cooperation and Development countries. Int J Qual Health Care. 2015;27(6):443-50. doi: http://doi. org/10.1093/intqhc/mzv074

27. Giraldo-Osorio A. Instrumentos que valoran las funciones de la atención primaria de salud: perspectiva de los usuarios. Rev Salud Publica (Bogotá). 2018;20(4):498-504. doi: http:/ /doi.org/10.15446/ rsap.V20n4.60261

28. Essa SA, Kamel MH, Mohammad OI, Shawky Ahmad NM. Assessment of the participation of primary care services in national tuberculosis control program in Gharbia Governorate. Egypt J Chest Diss Tuberc. 2017;66(2):321-5.

29. Oliveira SAC, Ruffino-Netto A, Villa TCS, Vendramini SHF, Andrade RLP, Scatena LM. Health services in tuberculosis control: family focus and community orientation. Rev Latinoam Enferm. 2009;17(3):361-7.

30. Villa TCS, Ruffino-Netto A. Questionário para avaliação de desempenho de serviços de atenção básica no controle da tuberculose no Brasil. J Bras Pneumol. 2009;35(6):610-2.

Manuscrito recibido el 30 de junio del 2020. Aceptado para publicación, tras revisión, el 6 de octubre del 2020. 


\section{Evaluation of the first level of care for tuberculosis control in Buenos Aires, Argentina}

ABSTRACT Objectives. Evaluate primary health care functions from the perspective of patients with tuberculosis from slums in the city of Buenos Aires, Argentina.

Methods. Cross-sectional observational study with adult patients with tuberculosis (TB) and without TB (NoTB), living in slums (S) and outside them (NoS). Participants' perceptions were evaluated using the Primary Care Assessment Tool for users (abbreviated version), which measures four main domains (first contact, ongoing care, coordination with specialists, and comprehensiveness) and selected secondary domains. A Likert scale was used, ranging from "No, not at all" (1 point) to "Yes, definitely" (4 points). Scores $\geq 3$ were considered to indicate adequate performance of functions. Averages were calculated for each domain, as well as two overall scores: with and without secondary domains.

Results. 83 participants were included (20 TB-S, 21 TB-NoS, 19 NoTB-S, and 23 NoTB-NS). The evaluated functions were perceived as inadequate. The TB-S group gave the lowest overall scores, not reaching 3 points in any domain. There were no significant differences in domains or overall scores between groups. Participants with TB gave lower scores in all domains, except in family-centered care, where they gave a significantly higher score than NoTB participants. The overall score without secondary domains was lower for TB participants than for the NoTB groups.

Conclusions. According to the perception of participants with TB and without TB, primary health care functions are not satisfactory, either in slums or outside them.

Keywords $\quad$ Poverty areas; tuberculosis; quality of health care; primary health care; Argentina.

\section{Avaliação da atenção primária à saúde no controle da tuberculose em Buenos Aires, Argentina}

RESUMO Objetivos. Avaliar as funções da atenção primária à saúde da perspectiva de pacientes com tuberculose (TB) provenientes de comunidades desfavorecidas na cidade de Buenos Aires, Argentina.

Métodos. Estudo observacional transversal com pacientes adultos com ou sem TB (TB, NãoTB), residentes ou não de comunidades desfavorecidas (C, NãoC). Avaliamos as percepções dos participantes utilizando questionário Primary Care Assessment Tool-usuários (versão reduzida), que mede quatro dimensões principais (primeiro contato, longitudinalidade da atenção, coordenação entre serviços e integralidade), bem como algumas dimensões secundárias. Utilizamos uma escala de Likert de 4 pontos, variando de "definitivamente não" (1 ponto) a "definitivamente sim" (4 pontos). Considerou-se que pontuações $\geq 3$ indicavam o cumprimento adequado das funções. Calculamos as médias para cada domínio e duas pontuações globais: com e sem domínios secundários.

Resultados. Ao todo, 83 participantes foram incluídos no estudo (20 TB-C, 21 TB-NãoC, 19 NãoTB-C e 23 NãoTB-NãoC). As funções avaliadas foram percebidas como inadequadas. O grupo TB-C apresentou a pontuação global mais baixa, não alcançando 3 pontos em nenhum domínio. Não houve diferenças significativas entre os grupos nos domínios nem na pontuação global. Os participantes com TB deram pontuações mais baixas em todos os domínios, exceto no enfoque familiar, no qual a pontuação foi significativamente mais alta que a dos participantes sem TB; a pontuação global sem domínios secundários foi mais baixa nos participantes com TB que nos sem TB.

Conclusões. De acordo com as percepções dos participantes com e sem TB, as funções da atenção primária à saúde são insatisfatórias, tanto dentro como fora das comunidades desfavorecidas.

Palavras-chave Áreas de pobreza; tuberculose; qualidade da assistência à saúde; atenção primária à saúde; Argentina. 